\title{
Landnahmen, lokale Wissensordnungen und Widerständigkeiten in Amazonien
}

\author{
Maria Backhouse
}

Eingegangen: 10. September 2020 / Angenommen: 15. Oktober 2020

(C) Der/die Autor(en) 2020

Zusammenfassung Widerständige Praktiken und lokale Wissensordnungen von Indigenen, traditionellen Gemeinschaften und kleinbäuerlichen Bewegungen sind in Brasilien seit Jahrhunderten eng mit Auseinandersetzungen um Landzugangsund Landnutzungsrechten verbunden. In der brasilianischen Verfassung von 1988 werden die spezifischen Landrechte wie kollektive Landzugangsrechte traditioneller Gemeinschaften garantiert. Sie sind hart erkämpfte Errungenschaften eines Projekts, welches Brasilien als soziale Einheit sieht und auf eine zunehmende demokratische Teilhabe aller ausgerichtet ist. Diese Landrechte werden unter der Regierung des rechtsradikalen Präsidenten Jair Messias Bolsonaro infrage gestellt und unterhöhlt. Im vorliegenden Artikel wird in Anknüpfung an die Interpretationen der fortgesetzten ursprünglichen Akkumulation von Massimo De Angelis argumentiert, dass die Bolsonaro-Regierung eine Landnahme angestoßen hat, die einige Kontinuitäten zu den Landnahmen der 1970er Jahre unter der Militärdiktatur aufweist. Darin kommt die Idee eines „Kern-Brasiliens“ zum Ausdruck, dessen Fortschritt unter Ausschluss oder Assimilierung der ,rückständigen“ Gruppen und traditionellen Gemeinschaften vorangetrieben werden soll. Allerdings ist der Ausgang dieses Landnahmeprojekts ungewiss, denn es bilden sich Widerständigkeiten und breite Allianzen, die ,das Soziale“ verteidigen - auch im sozial-ökologischen Sinne für zukünftige Generationen.

Schlüsselwörter Politische Ökologie · Landkonflikte · Landzugang und -nutzung · Traditionelle Landrechte · Bolsonaros Umweltpolitik

Jun.-Prof. Dr. M. Backhouse ( $ه)$

Institut für Soziologie, Friedrich-Schiller-Universität Jena, Bachstr. 18k, 07743 Jena, Deutschland

E-Mail: maria.backhouse@uni-jena.de 


\section{Land grab, local knowledge and resistance in Amazonia}

Abstract In Brazil, diverse practices of resistance and local knowledge of indigenous, traditional communities and peasant movements have been linked to conflicts around land access and land use for centuries. Brazil's 1988 constitution generally guarantees specific land use rights such as collective land rights for traditional communities. They represent hard-won victories of a project that regards Brazil as a social entity and is oriented toward increasing democratic inclusion of the whole society. Under the administration of far-right president Jair Messias Bolsonaro, these land rights are being challenged and undermined. Proceeding from Massimo De Angelis' interpretation of primitive accumulation, this paper argues that the Bolsonaro administration has initiated a process of land grabbing that exhibits continuities with the private appropriation of land occurring under the country's 1970s military dictatorship. One central feature in this context is the idea of a 'core Brazil', whose progress relies on the exclusion or assimilation of the 'backward' groups and traditional communities. However, the outcome of this land grab is uncertain, as we are currently witnessing new forms of resistance and alliances between diverse actors that go far beyond the local sites of conflict around land grabbing. Their struggle is in defense of 'the social' in the social-ecological sense, not least on behalf of future generations.

Keywords Political ecology · Land conflicts · Land access and use · Traditional land rights $\cdot$ Bolsonaro's environmental policy

In der Amazonasregion wüten Feuer und die Abholzungsrate steigt. Anfang September 2020 zählte das Weltrauminstitut Instituto Nacional de Pesquisas Espaciais (INPE) etwa 1000 Feuer in der Amazonasregion, im August desselben Jahres waren es 30.000 (Escobar et al. 2020). Die Abholzungsrate im Juli 2020 stieg im Vergleich zum Vorjahr 2019 um 34\% und im Vergleich zum Jahr 2018 um 50\% (Escobar et al. 2020). Diese Zerstörung der Natur ist untrennbar verbunden mit der Zerstörung der Lebensgrundlage von Indigenen und von heterogenen kleinbäuerlichen und traditionellen Gemeinschaften. Menschenrechtsbeobachter*innen stellen im Kontext der ökologischen Zerstörung einen großen Anstieg in historischem Ausmaß an gewaltsamen Angriffen auf indigene, traditionelle oder kleinbäuerliche Gemeinschaften fest, die im Zusammenhang stehen mit illegalem Holzeinschlag, Goldschürfen oder Landraub (CPT 2020). Die illegale Abholzung in indigenen Gebieten hat sich bereits in den ersten vier Monaten im Jahr 2020 im Vergleich zum Vorjahr vervierfacht (Jürgens 2020).

Die Gewalt und Zerstörung wachsen erkennbar seit der widerrechtlichen Amtsenthebung von Präsidentin Dilma Rousseff im Jahr 2016 (Canuto et al. 2020, S. 102). Mit der Wahl des rechtsradikalen Präsidenten Jair Messias Bolsonaro im Jahr 2018 nehmen sie sprunghaft zu (Canuto et al. 2020, S. 102). Bereits im Wahlkampf hatte er angekündigt, die Amazonasregion ökonomisch durch die Agrarindustrie und den Bergbau zu erschließen. Dieses Vorhaben verknüpft er mit verbalen Attacken gegen Indigene, traditionelle Gemeinschaften, kleinbäuerliche Bewegungen, 
Umweltschutzorganisationen und alle, die diese heterogenen, sozialen Bewegungen unterstützen. Berühmte Aussprüche bereits vor seiner Wahl waren: „Nicht ein Zentimeter wird mehr als indigenes Reservat demarkiert werden“ oder „2019 werden wir das indigene Reservat Raposa Serra do Sol zerlegen. Wir werden allen Ranchern Waffen geben“ (de Abreu und Russau 2020). Ähnlich werden auch die Anliegen der Landlosenbewegung Movimento dos Trabalhadores sem Terra (MST) und traditionelle Gemeinschaften wie die Quilombos, die auf entflohene Sklaven zurückgehen, attackiert. Entsprechend bestärkt fühlen sich Großgrundbesitzer (,fazendeiros"), Holzkonzessionäre, Landspekulanten oder Goldschürfer, was sich in der wachsenden Gewalt und Zerstörung widerspiegelt ${ }^{1}$.

Im vorliegenden Artikel wird in Anknüpfung an die Interpretationen der fortgesetzten ursprünglichen Akkumulation von Massimo De Angelis argumentiert, dass die Bolsonaro-Regierung eine Landnahme angestoßen hat, die einige Kontinuitäten zu den Landnahmen der 1970er Jahre unter der Militärdiktatur aufweist. Neu ist, dass nun ausdrücklich bereits erkämpfte indigene, traditionelle oder kleinbäuerliche Landrechte bedroht werden, die mit heterogenen lokalen Wissensordnungen der Land- und Waldressourcennutzung verwoben sind und sich als effektive Barrieren gegen Waldzerstörung und Vertreibung erwiesen haben.

Mit dem Artikel werden zwei Ziele verfolgt: Erstens soll gezeigt werden, wie bedroht indigene, kleinbäuerliche und traditionelle Landrechte sind, die eng verbunden sind mit heterogenen lokalen Wissensordnungen. Damit wird die Diskussion des Schwerpunktheftes über emanzipatorische Entwürfe des Sozialen in Brasilien, das auf die Anerkennung von Differenz und demokratischer Teilhabe aller ausgerichtet ist (siehe Einleitungsartikel von Benjamin Bunk und Emil Sobottka), ergänzt um die Analyse des zerstörerischen Gegenprojekts, wie es von der Bolsonaro-Regierung vorangetrieben wird. Darin kommt die Idee eines ,fortschrittlichen (Kern-)Brasiliens“ zum Ausdruck, dessen Fortschritt unter Ausschluss oder Assimilierung der „rückständigen“ Gruppen und traditionellen Gemeinschaften vorangetrieben werden soll. Zweitens wird begründet, warum ein flexibles Verständnis der Landnahme als Variante einer fortgesetzten ursprünglichen Akkumulation hilfreich ist, um diese aktuellen gesellschaftlichen Auseinandersetzungen zu analysieren und mit einer Zeitdiagnose zu verbinden.

Der Artikel ist folgendermaßen aufgebaut: Im folgenden Abschnitt wird anknüpfend an die politische Ökologie der Analyserahmen aufgespannt. Im dritten Abschnitt werden die Landnahmen während der Militärdiktatur und die widersprüchlichen Dynamiken während der Regierungsphase der Arbeiterpartei Partido dos Trabalhadores (PT) unter Luiz Inácio „Lula“ da Silva (2003-2010) und Dilma Rousseff (2011-2016) beschrieben. Im vierten Abschnitt wird das Projekt der Landnahme in Amazonien der Bolsonaro-Administration thematisiert. Da die Dynamiken aktuell

\footnotetext{
1 Landreformprojekte und die Territorien indigener und traditioneller Gemeinschaften werden in ganz Brasilien angegriffen. Ein besonders drastischer Fall, der international für Aufsehen sorgt, ist etwa die drohende Vertreibung von 450 Familien in der Landarbeitersiedlung Campo Grande in Zentralbrasilien (Bundesstaat Minas Gerais). Diese lebten seit 20 Jahren auf dem Land eines bankrottgegangenen Großgrundbesitzers (vgl. Schenk und Kliver 2020).
} 
stattfinden, sind diese Abschnitte deskriptiv und basieren auf der Auswertung von Zeitungsartikeln und Berichten.

\section{Klärung der Untersuchungsperspektive: Land, lokale Wissensordnungen und Landnahme}

Aus der Perspektive der politischen Ökologie ist Land Ausdruck des dialektischen Wechselverhältnisses zwischen Natur und Gesellschaft (Görg 2003): Menschen müssen Land bearbeiten, um sich ernähren zu können. Dabei werden sie von natürlichen Stofflichkeiten (z.B. Bodenqualität) beeinflusst. Wie aber Landzugang und -nutzung strukturiert sind, ist gesellschaftlich vermittelt, d.h. sie sind von historischen, ökonomischen, politischen und kulturellen Macht- und Herrschaftsverhältnissen durchdrungen. Diese Machtverhältnisse schreiben sich entlang von sozialen Ungleichheitsverhältnissen zwischen Regionen des Globalen Südens und Nordens und innerhalb Regionen entlang der Kategorien wie Klasse, Geschlecht, Ethnizität oder Alter in die Landzugangs- und Landnutzungsverhältnisse ein (Agarwal 1998). Sie sorgen dafür, dass auch Wissen und Praktiken der Landnutzung als modern oder veraltet auf- oder abgewertet werden. Denn Landzugang und Landnutzung sind mit lokalen Wissensordnungen verwoben. Im vorliegenden Kapitel sind mit lokalen Wissensordnungen heterogene kleinbäuerliche und traditionelle Landnutzungsformen gemeint, die in Brasilien, wie unten erklärt, komplementär oder als Gegenstück zur großflächigen Plantage (und später agrarindustrielle Monokultur) entstanden sind und immer wieder von Landnahmen bedroht sind. Sie sind untrennbar mit Kulturen, Traditionen und Weltanschauungen verbunden. Gleichzeitig sind sie positiver Bezugspunkt für soziale ländliche Bewegungen, die sich seit Jahrzehnten für eine heterogene ökologisch und sozial verträgliche Landwirtschaft einsetzen. Kleinbäuerlich und traditionell genutztes Land ${ }^{2}$ schließt im Amazonasgebiet spezifische Landnutzungsformen wie Wanderackerbau (z. B. für die Maniokproduktion), Agroforstsysteme sowie Sammel- und Jagdaktivitäten ein. Lokale kleinbäuerliche Wissensordnungen fasse ich als situiert und dynamisch auf (Escobar 1996; Haraway 1988). Es ist keineswegs klar von agrarwissenschaftlichem Wissen abgrenzbar, mit dem es z. B. über Förderprogramme, agrartechnische Beratung oder Ackerbauschulen schon lange in einem wechselseitigen Austauschverhältnis steht. Trotzdem behalte ich aber hier die vereinfachte Gegenüberstellung zwischen lokalen Wissensordnungen und Agrarindustrie bzw. Agrobusiness bei, da ich den Fokus auf aktuelle gesellschaftliche Auseinandersetzungen um Landnahmestrategien setze, die ausdrücklich eine Homogenisierungsstrategie verfolgen: nämlich die agrarindustrielle Erschließung Amazoniens, was letztlich die Zerstörung oder Assimilierung der heterogenen Kulturen lokaler Landnutzung bedeutet.

\footnotetext{
2 In Brasilien ist oft statt von Land von Territorien und territorialen Rechten die Rede, da damit in den Blick rückt, dass sich verschiedene territoriale Land- und Waldnutzungsweisen überlappen können. Ich denke diesen räumlichen Aspekt mit, bleibe aber der Einfachheit halber bei Landzugang und Landnutzung.
} 
Mit dem Begriff der Landnahme ${ }^{3}$ ist die Einhegung von kollektiv oder kleinbäuerlich genutztem Land für die Ausweitung von agrarindustriell produzierten Monokulturen oder Bergbau gemeint. Die Landnahme verstehe ich als eine Variante einer fortgesetzten ursprünglichen Akkumulation. Damit knüpfe ich an Interpretationen an, die „Die sogenannte ursprüngliche Akkumulation“ von Karl Marx (2005) nicht als Beschreibung einer abgeschlossenen historischen Phase des Übergangs vom Feudalismus zum Kapitalismus, sondern als Formierungsmoment des Kapitals selbst begreifen, das ,der Entstehung und Reproduktion des Kapitalismus inhärent ist“ (Alnasseri 2003, S. 133)4. Diese Rezeptionslinie geht auf Rosa Luxemburg (1923) zurück. Der Grundgedanke ist, dass der Kapitalismus auf ein nichtkapitalistisches Außen zurückgreifen kann, um sich zu reproduzieren. Mit dem nichtkapitalistischen Außen sind soziale Milieus und Beziehungen gemeint, die nicht von kapitalistischen Besitz- und Lohnverhältnissen durchdrungen sind. Sie können außerhalb (z. B. unkontaktierte indigene Völker in Amazonien) und innerhalb kapitalistischer Verhältnisse (z. B. sogenannte Hausfrauenarbeit) verortet sein. Im Kontext von Landnahmen können auch kapitalistische mit nichtkapitalistischen Verhältnissen neu kombiniert werden (Werlhof et al. 1988); ein Beispiel sind Landarbeiter*innen, die auf Plantagen zu Niedriglöhnen unter ihren Reproduktionskosten beschäftigt werden können, weil sie sich über die Subsistenzlandwirtschaft selbst reproduzieren (Wood 1983; Meillassoux 1978).

Um die politische Dimension des Konzepts für die Analyse zu stärken, knüpfe ich an die Marx-Interpretation von Massimo De Angelis (2001) an. Demzufolge ist die Trennung der Produzent*innen von ihren Produktionsmitteln das entscheidende Moment der sogenannten ursprünglichen Akkumulation (De Angelis 2001). Denn erst mit der Trennung wird das spezifische, kapitalistische Verhältnis hergestellt: die Kapitalbesitzer*innen auf der einen und doppelt freie Arbeiter*innen (persönlich frei und frei von Produktionsmitteln) auf der anderen Seite. Um sich reproduzieren (Nahrung, Kleidung, Unterkunft etc.) zu können, müssen die Arbeiter*innen ihre Arbeitskraft verkaufen (Kößler 2013, S. 27). Was also die Trennung im Rahmen der ursprünglichen Akkumulation von Trennungsprozessen im normalen Gang der Akkumulation unterscheidet, ist, dass sie kapitalistische Verhältnisse für eine bessere Mehrwertabschöpfung erstmals herstellt oder umfassend restrukturiert. Entscheidend für meine Analyse ist nun, dass diese Trennungsprozesse als umkämpft und kontingent aufgefasst werden (De Angelis 2001). Aus dieser Perspektive ist die fortgesetzte ursprüngliche Akkumulation nicht ökonomisch determiniert oder Aus-

\footnotetext{
3 Ich verwende den Begriff der Landnahme mit dieser Eingrenzung anders als Klaus Dörre, der mit der Metapher der Landnahme eine Neuinterpretation der fortgesetzten ursprünglichen Akkumulation formuliert und entsprechend sämtliche gesellschaftliche Bereiche einschließt (vgl. Dörre 2009).

4 Einflussreiche Interpretationen der fortgesetzten ursprünglichen Akkumulation als erstmalige Durchsetzung oder Restrukturierung kapitalistischer Produktions- und Besitzverhältnisse sind beispielsweise das Konzept der Akkumulation durch Enteignung von Harvey (2005); die der Einhegung öffentlicher Güter (Commons) von De Angelis (2001); der Inwertsetzung natürlicher Ressourcen von Görg (2004); die innere Landnahme in Form von Prekarisierung von Lutz (1984) und Dörre (2009); der fortgesetzten ursprünglichen Akkumulation als Ausbeutung des Sorgesektors von Werlhof et al. (1988); der brasilianischen Debatte um die kapitalistische ,frontier“ in Amazonien von de Martins (2009), Foweraker (1981) und Velho (1979) oder des Werttransfers von den Peripherien des globalen Südens in die Zentren des globalen Nordens von Wallerstein (1986).
} 
druck eines linear fortschreitenden Ökonomisierungsprozesses. Vielmehr rücken die Akteur*innen und ihre Kämpfe in den Mittelpunkt der Analyse, die den Verlauf von Trennungsprozessen im Kontext von Landnahmen unterstützen, verändern oder verhindern können. Dieser Fokus auf die politische Dimension der Trennungsprozesse ermöglicht, Prozesse der fortgesetzten ursprünglichen Akkumulation als historisch spezifisch und ergebnisoffen zu begreifen. Um diesen Aspekt zu vertiefen, nimmt De Angelis eine Erweiterung in Anschluss an Karl Polanyis (1978) Konzept der „Doppelbewegung" zwischen der Verselbstständigung des Markts und gesellschaftliche Gegenbewegungen zu seiner Regulierung vor: Eine Trennung findet demzufolge auch statt, wenn soziale Barrieren, die von sozialen Bewegungen erfolgreich gegen die ungehinderte Ausbreitung der Marktlogik durchgesetzt worden sind, erneut unter den Druck von kapitalistischen Landnahmen geraten (De Angelis 2001, S. 13).

Allerdings sind Trennungsprozesse im Sinne einer fortgesetzten ursprünglichen Akkumulation nicht nur auf die Herstellung von Lohnarbeit oder ihre Prekarisierung nach westlichem Vorbild beschränkt, sondern nehmen unterschiedliche Formen an. Sie schließt auch Kolonialismus und Sklaverei mit ein, wie anti- und postkoloniale Theoretiker*innen gezeigt haben (Quijano 2016; Wallerstein 1986; Fanon 1981). Demzufolge trug der Kolonialismus mit der immensen Ausbeutung von menschlichen und natürlichen Ressourcen entscheidend zu der Herausbildung des Kapitalismus bei. Denn die erstmalige Trennung etablierte die internationale Arbeitsteilung zwischen Globalem Süden und Globalem Norden und restrukturiert tiefgreifend die kolonisierten Gesellschaften (De Angelis 2001, S. 11 f.). Sklaverei und Schuldknechtschaftssysteme sind somit keine feudalen Überbleibsel einer präkapitalistischen Zeit, sondern vielmehr ein wichtiger Bestandteil des Kapitalismus. Je nach lokalen und transregionalen kolonialen Machtverhältnissen bildeten sich im Trennungsprozess spezifische kapitalistische Verhältnisse heraus, die auch lange nach dem formalen Ende des Kolonialismus etwa über strukturelle und kulturelle Rassismen fortwirken (Quijano 2016). Die Kontinuität kolonialer Verhältnisse gilt es in der Analyse von aktuellen Landnahmen zu berücksichtigen, denn ungleiche Landzugangs- und Landnutzungsverhältnisse entlang der Kategorien Geschlecht, Ethnizität und Klasse wurden in vielen Ländern des Globalen Südens in der heute bekannten Form erst während des Kolonialismus angelegt und strukturierten auch spätere gesellschaftliche Umwälzungen im Rahmen der grünen Revolution in den 1970er und Strukturanpassungsmaßnahmen in den 1980er Jahren (Whitehead 2016). Gleichzeitig waren diese kolonialen Landnahmen auch mit einer Abwertung lokaler Wissensordnungen als unterentwickelt oder irrational verbunden (Quijano 2016).

Damit wird auch die sprachlich-symbolische Ebene der Trennung angesprochen: Denn Trennungsprozesse betreffen nicht nur die materielle Ebene der Enteignung von Produktionsmitteln, sondern auch die kulturellen Praktiken und Wissensordnungen, die mit diesen spezifischen gesellschaftlichen Naturverhältnissen verwoben sind und tiefgreifend verändert werden. Zusätzlich stellt sich auch die Hegemoniefrage bzw. die Frage nach der Herstellung überwiegender gesellschaftlicher Zustimmung zu einem Projekt der Landnahme oder zu Widerspruch und Widerständigkeiten (Backhouse 2015).

Neben der erstmaligen oder vertieften Trennung ist ein weiteres definitorisches Merkmal der Landnahme, dass die Trennungsprozesse nicht durch die Wirkung des 
reinen Marktes, sondern über extraökonomische Methoden oder Mittel wie staatliche Förderprogramme, Gesetzesänderungen oder Gewalt hergestellt werden (De Angelis 2001, S. 9). Diese Methoden der Trennung wie Gewalt oder Gesetze sollten aber nicht überbewertet werden. Die Analyse muss vielmehr zeigen, dass über diese Methoden der Trennung soziale Verhältnisse tiefgreifend restrukturiert werden. Die Untersuchung von gesellschaftlichen Dynamiken, die eine Landnahme vermuten lassen, erfordert vor diesem Hintergrund eine flexible und kontextspezifische Herangehensweise. Letztlich muss geklärt werden, ob eine erstmalige oder vertiefte Trennung im Sinne einer fortgesetzten ursprünglichen Akkumulation stattfindet. Dies erfordert eine historisch sensibilisierte Herangehensweise an die bestehenden Landzugangs- und Landnutzungsverhältnisse, eine Klärung der extraökonomischen Mittel der Landnahme ebenso wie die gesellschaftlichen Auseinandersetzungen um den Trennungsprozess.

\section{Landnahmen und Widerständigkeiten in Amazonien}

In Brasilien kontrollieren knapp 1\% der Landbesitzer*innen etwa $45 \%$ der landwirtschaftlichen Nutzflächen (Viehweiden und Ackerbau; Oxfam Brazil 2019) ${ }^{5}$. Die hohe Landkonzentration drückt bis heute unterschiedliche Landnutzungssysteme aus: Während die kleinbäuerliche Landwirtschaft in Brasilien einen beachtlichen Teil der regional konsumierten Grundnahrungsmittel (z.B. $80 \%$ des Manioks) ${ }^{6}$ produziert, konzentriert die agrarindustrielle Exportproduktion von Soja, Zucker und Rindfleisch den Großteil der Fläche auf sich.

Diese große Konzentration von Land wurde bereits in den Kolonialzeiten etabliert $^{7}$ und ist eine der historischen Ursachen für die eklatanten sozialen Ungleichheiten in Brasilien. Mit Ausnahme von Südbrasilien, wo europäische kleinbäuerliche Familien angesiedelt wurden ${ }^{8}$, entstanden in Zentral-, Nordost- oder Nordbrasilien kleinbäuerliche Strukturen auf den Subsistenzflächen von Sklaven, widerständigen entflohenen Sklaven (,quilombolas“) oder Landlosen in Grenzgebieten zwischen Großgrundbesitztümern (,fazendas“), auf an den Staat zurückgefallenen Flächen bzw. Staatsland (,terras devolutas“), in noch nicht erschlossenen Regionen oder in Ungunstlagen. Sie produzierten schon zu Kolonialzeiten hauptsächlich die regional konsumierten Nahrungsmittel wie Maniok, Bohnen oder Reis. Die kleinbäuerliche Landwirtschaft ist somit nicht der Vorläufer der großflächigen Landwirtschaft,

\footnotetext{
5 Diese Zahlen sind Schätzwerte auf der Basis von Daten des Agrarzensus IBGE (Instituto Brasileiro de Geografia e Estatistica) aus den Jahren 2006 und 2009, vgl. www.ibge.gov.br (letzter Aufruf: 01.09.2020).

6 Vgl. dazu den Agrarzensus von 2017 zur Kleinbäuerlichen Familie: www.ibge.gov.br (letzter Aufruf: 01.09.2020).

7 Zum kolonialen Seismaria-System, das bereits den großflächigen Landraub etablierte und nach der Unabhängigkeit Brasiliens vom Landgesetz (Lei de Terras; Gesetz Nr. 601) aus dem Jahr 1850 abgelöst wurde (vgl. Torres 2018; Bröckelmann-Simon 1994, S. 40-47; Assunção 1993, S. 109-113).

8 Die europäischen Einwander*innen aus Deutschland und Italien wurden in Gebieten angesiedelt, die für den Großgrundbesitz uninteressant waren. Auch in dieser Region entstanden Konflikte um Landnutzungsrechte, weil die Bodenbesitzverhältnisse nicht reguliert wurden und der Bedarf an Land durch die wachsende Bevölkerung zunahm (vgl. Bröckelmann-Simon 1994, S. 48-65).
} 
sondern entstand bereits in der Kolonialzeit dort, wo der Großgrundbesitz Lücken ließ oder in der Krise war (Treccani 1998, S. 60). Bis heute sind viele Kleinbauern und Kleinbäuerinnen insbesondere in den peripheren Regionen Brasiliens im Norden oder Nordosten nicht die Eigentümer*innen des bewirtschafteten Landes, sondern nur Landbesitzer mit Gewohnheitsrechten (,posseiros“). Der Widerspruch zwischen Großgrundbesitz und kleinbäuerliche Wirtschaft führt seit Kolonialzeiten zu Konflikten zwischen Plantagenbesitzern und diversen kleinbäuerlichen Bewegungen. Die Geschichten der Landnahmen waren von Anfang an auch Geschichten der Widerständigkeiten gegen Landraub und Vertreibung mit unterschiedlichem Ausgang 9 .

\subsection{Landnahmen während der Militärdiktatur}

Das Rechtsgebiet Amazonien (Amazônia Legal) setzt sich aus neun Bundesstaaten zusammen und umfasst etwa 59\% des brasilianischen Nationalterritoriums. Amazonien steht seit Kolonialzeiten immer wieder im Fokus von Erschließungsprojekten. Doch erst unter der Militärdiktatur (1964-1985) wurde eine Landnahme in vorher unbekanntem Ausmaß in Gang gesetzt. Über einen Zeitraum von etwa zwei Jahrzehnten wurden auf der Grundlage einer autoritären Entwicklungsideologie (,,desenvolvimentismo“) verschiedene Erschließungsprojekte initiiert (Neuburger 2002, S. 72), mit denen unterschiedliche geostrategische, macht- und entwicklungspolitische Ziele verfolgt wurden:

- Die Landkonflikte im Süden und Nordosten Brasiliens sollten entschärft werden, indem die Landlosen in der angeblich menschenleeren Amazonasregion angesiedelt werden sollten. Die durch den Militärputsch verhinderte Agrarreform wurde auf diese Weise auf eine Agrarkolonisierung Amazoniens verlagert (de Oliveira 2005, S. 72; Treccani 1998, S. 98).

- Das eigene Klientel und Bündnispartner aus privatwirtschaftlichen Kreisen und Eliten der südöstlichen Metropolen Brasiliens sollten über großzügige fiskalische Anreize und die Einrichtung der Freihandelszone Manaus zu Investitionen angeregt werden (Coy 1988, 33 ff.).

- Das von der Militärdiktatur unter Getúlio Vargas bereits in den 1940er Jahren initiierte Projekt, Amazonien nationalstaatlich zu integrieren, sollte nun verwirklicht werden (Abbrex Jr 2005, S. 32-37). Über die infrastrukturelle Erschließung, Besiedlung und Inwertsetzung der Region sollten geopolitische Interessen zur Absicherung des nationalstaatlichen Territoriums und des direkten Zugriffs auf die Ressourcen (Land, Holz und Bodenschätze) durch die Zentralregierung abgesichert werden. Ziel war dabei auch die Verhinderung der Vereinnahmung der Region durch ausländische Kräfte und Interessen (Schmink und Wood 1992, $58 \mathrm{f}$.). Diese nationalistische Argumentation findet, wie sich unter der aktuellen Bolsonaro-Regierung zeigt, bis heute Anklang der brasilianischen Gesellschaft.

\footnotetext{
$9 \mathrm{Zu}$ den Geschichten der Aufstände und Widerständigkeiten der diversen kleinbäuerlichen Akteure in ganz Brasilien (vgl. Almeida 2008; Assunção 1993; Bröckelmann-Simon 1994).
} 
Zwar förderten die staatlichen Entwicklungsprogramme die kleinbäuerliche Landwirtschaft durch Agrarreform-Siedlungsprojekte (,,assentamentos"), im Fokus stand aber der Privatsektor und die Umsetzung einer wachstums- und exportorientierten Modernisierungsstrategie (Neuburger 2002, S. 73). Die Siedlungsprojekte auf der einen Seite und fiskalische Anreize für Investitionen und Bodenspekulation auf der anderen Seite sowie das Ignorieren der Landrechte der in der Region lebenden amazonischen Bevölkerung schürten diverse Konfliktkonstellationen auch zwischen den kleinbäuerlichen Interessensgruppen (Schmink und Wood 1992; Schönenberg 1993).

Kommentator*innen beschrieben dieses große staatlich initiierte Landnahmeprojekt als ,greatest land rush since the settling of the American West“ (Washington Post, zitiert nach Browder et al. 2008, S. 1474). Doch verglichen mit dem USamerikanischen Westen scheiterte dieses Projekt letztlich an der ökomischen Krise Brasiliens (Cleary 1993) und an den Widerständigkeiten unterschiedlicher amazonischer Gruppen und Bewegungen (Schmink und Wood 1992). Weltweit berühmt wurde etwa der Kampf der Gewerkschaft der Kautschukzapfer*innen unter der Leitung von Chico Mendes für den Schutz ihrer Sammelgebiete.

\subsection{Landnahmen während der demokratischen Regierungen}

Nach dem Ende der Militärdiktatur und der Verabschiedung der progressiven brasilianischen Verfassung aus dem Jahr 1988 wendete sich das Blatt. In den 1990er Jahren setzte sich die nachhaltige Entwicklung als Leitziel der Regionalplanung für das Amazonasgebiet durch. Bekräftigt wurde diese Entwicklungsperspektive im Jahr 1992 mit der UN-Nachhaltigkeitskonferenz in Rio de Janeiro. Es wurden neue kollektive Eigentumstitel auf der Basis der neuen brasilianischen Verfassung geschaffen und mit dem ökologischen Ziel des Waldschutzes verbunden: Die Demarkierung von Naturschutzgebieten, Extraktionsreservaten für Kautschuk- oder Paranuss-Sammler*innen (Reservas Extrativistas), Territorien indigener Völker und traditioneller Gemeinschaften wie die Nachfahren widerständiger Sklaven (Quilombos) sollte eine ressourcenschonende Nutzung sicherstellen (Neuburger 2002, S. 77). Diese Landzugangsrechte sind mit spezifischen Landnutzungen verbunden: Kollektiv tituliertes Land traditioneller Gemeinschaften darf nur traditionell genutzt werden und ist dauerhaft dem Landmarkt und der Agrarindustrie entzogen. Traditionell heißt in diesem Zusammenhang, dass nur schonende Ressourcen- und Landnutzungsformen zulässig sind, die sich in dem jeweiligen historischen, regionalen und kulturellen Kontext herausgebildet haben (Almeida 2010a). Für den Privatbesitz schreibt das Waldgesetz vor, dass $80 \%$ der Fläche bewaldet sein oder entsprechend wieder aufgeforstet werden müssen. Auch die Landlosenbewegung MST wurde zunehmend zu einer wichtigen politischen Kraft in der Amazonasregion und begann in ganz Brasilien ein agrarökologisches Gegenmodell zum Großgrundbesitz und der Agrarindustrie zu entwickeln. Eine zentrale Aktionsform ist die Landbesetzung von geraubten Landgütern und darüber einen juristischen Prozess anzustoßen, über den am Ende entschieden wird, ob das Land umverteilt wird (Stedil und Fernandes 1999). Hervorzuheben ist, dass der MST seine Kämpfe um Landrechte mit emanzipatorischen Bildungsan- 
sätzen verbindet ${ }^{10}$. Gemeinsam ist den hier skizzierten sozialen Bewegungen, dass sie zu einer Aufwertung lokaler Wissensordnungen beitragen.

Dieses nachhaltige Entwicklungsprojekt war aber von Anfang an widersprüchlich: Zum einen wurden in diesem Rahmen marktbasierte Umweltschutzmaßnahmen etabliert, die Landnahmen auslösen können; z. B. wenn Biodiesel auf Palmölbasis zur Ausweitung von Plantagen führt, die neue Verdrängungsdynamiken entfalten (Backhouse 2015). Zum anderen stand das Ziel des Waldschutzes im Widerstreit mit dem Ziel der ökonomischen Erschließung, was ebenfalls widersprüchliche Effekte zur Folge hatte: Die Abholzungsrate ging zwischen 2004 und 2014 um $82 \%$ zurück $^{11}$, was ein großer Erfolg war und u.a. auf die Demarkierung von Gebieten indigener Völker oder traditioneller Gemeinschaften zurückgeführt werden kann. Denn in diesen Gebieten wird der Wald nachweislich geschützt (Torres 2018). Die Erfolge bei der Anerkennung der Landrechte von Territorien traditioneller Gemeinschaften und Agrarreformsiedlungen sollten jedoch nicht darüber hinwegtäuschen, dass sie auf große Widerstände ländlicher Eliten, des Agrobusiness oder von Holzkonzessionären gestoßen sind (Almeida 2010b; Torres 2005). Gleichzeitig wurden diese Landrechte von der brasilianischen Agrarlobby über Gesetzesinitiativen oder Kampagnen gegen angeblich illegitime Sonderrechte, die einer Entwicklung Brasiliens entgegenstehen würden, infrage gestellt (Almeida 2010b). Das Projekt der Landumverteilung und der Agrarreform rückten bereits während der Regierungszeit der Arbeiterpartei PT zunehmend in den Hintergrund. Insbesondere unter der Regierung von Dilma Rousseff war die Anzahl der Demarkierungen und Titulierungen von Agrarreformsiedlungen, indigenen und traditionellen Gebieten verglichen mit Lula und seinem konservativen Amtsvorgänger Fernando Henrique Cardoso (1995-2003) rückläufig ${ }^{12}$. Gleichzeitig nahmen die extensiven Viehweiden, der Sojaanbau (Torres und Branford 2018), die Vergabe von Bergbaukonzessionen (Maerba et al. 2012), der Ausbau von Megainfrastrukturprojekte wie Staudämme für die Stromgenerierung und Wasserstraßennetze (Almeida und Carvalho 2009) weiterhin zu.

Landzugang und -nutzung blieben somit Gegenstand gewaltsamer Konflikte, die verschärft wurden, weil die Landzugangsrechte bis heute vielfach ungeklärt sind. Expert*innen gehen davon aus, dass bei $50 \%$ der Fläche die Landbesitzverhältnisse ungeklärt sind (Brito und Barreto 2009). Aufgrund der Praxis des Landraubs über Urkundenfälschung (,grilagem“) ${ }^{13}$ werden wesentlich mehr Landbesitzansprü-

\footnotetext{
10 Diese emanzipatorischen Bildungsansätze entstanden im Kontext der Befreiungstheologie und Befreiungspädagogik. Auch bei den anderen genannten sozialen Bewegungen spielten Organisationen wie die katholische Landpastorale Comissão Pastoral da Terra (CPT) oder die NGO Federação de Órgãos para Assistência Social e Educacional (FASE) eine wichtige Rolle. Keine Bewegung hat aber in vergleichbarem Ausmaß eigene Bildungsinstitutionen wie die Bildungseinrichtung Florestan Fernandes gegründet. Zu der Entwicklung der emanzipatorischen Bildungspraxis der Landlosenbewegung MST: Bunk 2018.

11 Siehe dazu die Zahlen und Tabellen des Weltrauminstituts INPE seit 1988: http://www.inpe.br/noticias/ noticia.php?Cod_Noticia=5294 (zuletzt aufgerufen am 04.09.2020).

12 Siehe dazu die Übersicht der NGO Instituto Socioambiental (ISA) auf der Basis der Daten der Agrarreformbehörde Instituto Nacional de Colonização e Reforma Agrária (INCRA; vgl. Souza 2016).

13 Der Begriff „grilagem“ beschreibt die Praxis des Landraubs zu Kolonialzeiten: Die gefälschte Besitzurkunde wurde zusammen mit einer Grille in eine Schublade gesteckt. Das Sekret der Grille hat das Papier alt und echt aussehen lassen, siehe zu den modernen Praktiken des Landraubs: Torres (2018).
} 
che gestellt als Landflächen vorhanden sind (Torres 2018). Der amazonische Bundesstaat Pará, der 3,5-mal so groß wie Deutschland ist, könnte vier Stockwerke bilden, wenn alle Landansprüche in den Grundbuchämtern zusammengerechnet werden. Laut Landforscher Maurício Torres (2018) führten bisher alle Gesetzesinitiativen, dieses Konfliktpotenzial über die Titulierung von Privatbesitz zu beheben, durchgehend zu einer Legalisierung und Institutionalisierung von Landraub. Das Programm Terra Legal aus dem Jahr 2009, das unter der Lula-Regierung umgesetzt wurde, war keine Ausnahme (Torres 2018). Ziel des Programms war ursprünglich, die Landrechte von kleinbäuerlichen Familien über private Eigentumstitel zu regulieren, um Rechtssicherheit zu schaffen. Dafür sollten u. a. alle Landflächen bis zu maximal vier Landeinheiten (,módulos fiscais“) ${ }^{14}$, die insgesamt maximal $1500 \mathrm{Ha}$ umfassen und vor dem Jahr 2004 besetzt worden sind, tituliert werden. Vorgesehen war ursprünglich, dass $85 \%$ der Begünstigten kleinbäuerliche Familien sind. Letztlich profitierten aber die Grileiros: Knapp 6\% der Antragstellenden besitzen demzufolge $63 \%$ der titulierten Fläche (Torres et al. 2017, S. 30). Mithilfe von Strohmännern („laranjas“), die für die Registrierung unterschiedlicher Parzellen von 1500Ha großer Flächen von mehreren $10.000 \mathrm{Ha}$ eingesetzt wurden, gelang die rechtliche Absicherung des Landraubs im großen Stil.

\section{Das neue alte Landnahmeprojekt unter Jair Messias Bolsonaro}

Seit der Redemokratisierung Brasiliens in den 1980er Jahren wurde kein Projekt der ökonomischen Erschließung Amazoniens in dieser Größenordnung und Radikalität initiiert wie nun unter Bolsonaro. Dies hat machtstrategische Gründe: Laut der Journalistin Eliane Brum (2020) ist die Erschließung Amazoniens der kleinste gemeinsame Nenner, um konkurrierende konservative und rechte politische Kräfte zu vereinen (Brum 2020, S. 109).

Eine Kontinuität zu den Erschließungszielen der Militärs der 1970er Jahre ist die Reaktivierung des nationalistischen Projekts, Amazonien ökonomisch zu ,integrieren, um [es] nicht [an ausländische Mächte] zu verlieren (,Integrar para não entregar')“". Damit werden nach wie vor große Teile der brasilianischen Gesellschaft angesprochen. Interventionen seitens ausländischer Regierungen und internationaler Umweltschutzorganisationen werden als kolonialistisch empfunden, wie die Reaktionen auf den Tweet von Emmanuel Macron „Unser Haus brennt ... Der Regenwald in Amazonas ... steht in Flammen“ im Zusammenhang mit den Feuern in Amazonien im August 2019 verdeutlichten ${ }^{15}$.

Neu an dem Landnahmeprojekt Bolsonaros ist, dass die indigenen, traditionellen und kleinbäuerlichen Landrechte besonders im Fokus dieser vom Staat gestützten Erschließungspolitik stehen. Das heißt, auch sicher geglaubte Landrechte und bereits

\footnotetext{
14 „Módulos fiscais“ sind Steuereinheiten, die von der Agrarreformbehörde INCRA definiert werden und je nach Region in ihrer Größenordnung variieren.

15 Der Tweet von Emmanuel Macron wurde nicht nur von Bolsonaro, sondern auch von einem großen Teil der brasilianischen Gesellschaft zurückgewiesen. Sogar die linksliberale Zeitung Folha de São Paulo, die Bolsonaro meist kritisch gegenübersteht, springt ihm an dieser Stelle bei (vgl. Peters 2019).
} 
titulierte Gebiete stehen nun erneut zur Disposition. Damit wendet sich Bolsonaro ausdrücklich gegen die brasilianische Verfassung von 1988 und die Ratifizierung internationaler Normen wie das Übereinkommen der internationalen Arbeitsorganisation zu den Rechten indigener Völker (ILO 169). Hierbei geht es nicht nur um die Erschließung von Bodenschätzen und um Land für das Agrobusiness, sondern auch um die Rücknahme der gesetzlich festgeschriebenen Anerkennung heterogener Kulturen und Landnutzungspraktiken. Im Fadenkreuz stehen die Landlosenbewegungen, Indigene und Quilombolas in besonderer Weise. Gleich zu Beginn seines Amtes twitterte er: „Mehr als $15 \%$ des nationalen Territoriums ist als indigenes oder Quilombola-Land tituliert. Weniger als eine Million Personen leben dort, isoliert vom echten Brasilien, ausgebeutet und manipuliert von NGOs. Lasst uns diese Bürger integrieren und wie alle Brasilianer wertschätzen“ (Alessi 2019). Wie Brum verdeutlicht, nimmt Bolsonaro eine Verschiebung der Argumentation der alten Rechte vor. Diese argumentiert seit Jahrzehnten, dass die Indigenen von heute keine echten Indigenen mehr seien und entsprechend kein Anrecht mehr auf ihr Land hätten. Bolsonaro hingegen leugnet nicht, dass sie Indigene sind, sondern bietet ihnen an „Menschen wie wir“ zu werden. Die Landnahme legitimiert er als ein emanzipatorisches Projekt: Indigene sollen das Recht haben, ihr Land auszubeuten oder zu verkaufen „wie wir“ (Brum 2020, S. 109).

Es ist angemessen von einem Landnahmeprojekt $\mathrm{zu}$ sprechen, da es erst am Anfang steht und der Ausgang dieser großen Erschließung Amazoniens noch unklar ist. Die Daten zu den wachsenden Raten der Abholzung, Brände und Landkonflikte verdeutlichen aber, dass dieses Projekt schon längst in Gang gesetzt ist. Zentrale nichtökonomische Mittel der Landnahme sind hierbei erstens die verbalen Angriffe seitens des Präsidenten und seiner Kabinettsmitglieder, die den Grileiros Straffreiheit für Landraub suggerieren und soziale Bewegungen wie die Landlosenbewegung MST als terroristisch kriminalisieren.

Zweitens ist die Aushöhlung von staatlichen Institutionen, die für die Durchsetzung indigener, traditioneller und kleinbäuerlicher Landrechte verantwortlich sind, ein sehr effektives extraökonomisches Mittel der Landnahme. Darunter wird die Strategie verstanden, Leitungspositionen mit Vertretern aus dem Militär, der Polizei oder des Agrobusiness-Umfeldes zu besetzen oder die Institutionen mit Sparmaßnahmen oder Verschiebungen der Zuständigkeiten handlungsunfähig zu machen. Ein Beispiel ist der Umweltminister Ricardo de Aquino Salles, der den Wald- und Umweltschutz im Interesse des Agrobusiness von innen sabotiert. Seinem Ministerium wurde die Umweltschutzbehörde Instituto Brasileiro do Meio Ambiente e dos Recursos Naturais Renováveis (IBAMA) unterstellt, die u.a. für Bekämpfung der illegalen Abholzung zuständig und aufgrund der Sparmaßnahmen sowieso kaum mehr handlungsfähig ist. Ähnlich wurde auch die Leitungsposition der Indigenenbehörde Fundação Nacional do Índio (FUNAI) mit einem Mitglied der Agrarlobby im Parlament (,,bancada ruralista“) besetzt. Kritiker*innen befürchten, dass mit dieser Besetzung die ausstehenden Titulierungen indigener Gebiete paralysiert werden (ISA 2019).

Drittens sind Gesetzesinitiativen eine weitere klassische Methode der Landnahme, wie sie schon K. Marx beschrieben hat. Mit seinen Gesetzesinitiativen unterstreicht die Bolsonaro-Administration, wie ernst es ihr mit der Erschließung Amazoniens ist. 
Ein Beispiel ist der Gesetzesentwurf PL 191/2020 (Projeto de Lei) im Februar 2020, der Bergbau, Wasserkraft, industrielle Landwirtschaft sowie Exploration von Öl- und Erdgasvorkommen in indigenen Schutzgebieten legalisieren soll. Das Gesetz löste weltweite Proteste aus und wurde vorerst vom Präsidenten der Abgeordnetenkammer gestoppt. Eine Sonderkommission soll nun zur Analyse des Projekts eingerichtet werden.

Ein weiteres Beispiel ist die Gesetzesinitiative mit dem Namen provisorische Maßnahme MP 910 (Medida Provisória), die Bolsonaro noch kurz vor Weihnachten 2019 auf den Weg brachte und als „Gesetz für Landraub“ ebenfalls weltweite Proteste auslöste. Es sollte das Landtitulierungsprogramm Terra Legal (s. oben) ablösen und die Titulierung von Privatbesitz für noch größere Flächen - statt vier nun 15 Landeinheiten ohne Vorortkontrolle - weiter vereinfachen. Möglich sollte das für Flächen werden, die bis 2018 in Besitz genommen worden sind. Die Landkatastrierung sollte online erfolgen; ein Eigentumsnachweis wäre nicht erforderlich gewesen. Letztlich sollten ,die illegalen Landnahmen der jüngsten Vergangenheit, die meist durch illegale Brandrodung in die Wege geleitet werden, [...] durch Landtitelvergabe ... [im Nachhinein legalisiert werden], dies zu geringeren als den üblichen Marktpreisen“, fasst Christian Russau (2020) zusammen. Dabei ist bereits die gängige Praxis der digitalen Umweltregistrierung über das Cadastro Ambiental Rural (CAR) problematisch, da es ein sehr effektives Instrument der Legalisierung des Landraubs ist (Torres 2018). Wie Greenpeace am Fall des Indigenengebiets Ituna-Itatá zeigt, wird Land auch in indigenen Gebieten über das CAR registriert (Greenpeace 2020; Russau 2020). Zusätzlich ist das Gesetz ein klarer Vorstoß gegen Landreformprojekte. Denn auch die Landtitelüberschreibung in den Landreformsiedlungen soll vereinfacht werden, was einer beschleunigten Privatisierung von Land innerhalb von Agrarreformprojekten gleichkommt. Bisher können Landparzellen innerhalb von Landreformprojekten zwar vererbt aber - zumindest legal - nicht auf dem Landmarkt gehandelt werden (Russau 2020). Diese Gesetzesinitiative scheiterte an den großen Widerständen aus der brasilianischen Gesellschaft und dem Kongress. Die enthaltenen Ziele von $M P 910$ werden aber im Gesetzesentwurf $P L$ 2633 weiterverfolgt. Dieser wurde von dem Präsidenten der Abgeordnetenkammer für die Zeit nach der Corona-Pandemie vertagt. Zwar nimmt dieser Gesetzesentwurf im Vergleich zur MP 910 einige Einschränkungen vor, was die Größe (nur sechs statt 15 Landeinheiten) der zu legalisierenden Fläche angeht. In seiner Ausrichtung bleibt er gleich: Statt illegale Abholzung und Landraub strafrechtlich zu verfolgen, wird diese Praxis belohnt mit legalen Landtiteln.

Die wachsende Gewalt und die Kriminalisierung sozialer Bewegungen und NGOs führen bereits dazu, dass Landbesetzungen durch soziale Bewegungen wie der Landlosenbewegung MST, um widerrechtlichen Landraub rückgängig zu machen oder die Umverteilung von Land zu erzwingen, rückläufig sind (CPT 2020). Das bedeutet aber nicht, dass die Landnahmen der Bolsonaro-Regierung unwidersprochen bleiben. Allein im Jahr 2019 zählt die katholische Landpastorale CPT über 1301 Demonstrationen von über 240.000 Menschen (CPT 2020, S. 7). Über (inter)nationale Kampagnen versuchen die sozialen Bewegungen und ihre Unterstützer*innen zusätzlich internationalen Druck zu erzeugen. Die weltweite Mobilisierung gegen die Gesetzesinitiative MP 910 kann zumindest als Etappensieg eingestuft werden. 


\section{Schlussfolgerung}

Die Feuer und Abholzungsraten haben den Blick der Weltöffentlichkeit wieder auf Amazonien gelenkt. Im vorliegenden Kapitel wurde deutlich, dass die Waldzerstörung eng zusammenhängt mit Landnahmen, die eine über 500-jährige Geschichte haben. Diese zerstören nicht nur Natur, sondern führen auch zu tiefgreifenden Trennungsprozessen von diversen Akteuren von ihren Lebensgrundlagen. Das Landnahmeprojekt unter Bolsonaro steht in dieser Tradition. Neu ist, dass bereits erkämpfte Landrechte im Fokus der Landnahme stehen. In den Worten von De Angelis stehen somit soziale Barrieren gegen eine Durchkapitalisierung der amazonischen Region unter erneutem Druck. Neu ist aber auch, in welchem Ausmaß der Staat selbst zum Treiber dieser Landnahmen wird. Was vor Bolsonaro immerhin als Verbrechen galt, auch wenn es aufgrund von klientelistischen Netzwerken, schwacher Präsenz des Staates und großen Machtgefällen oft nicht geahndet wurde, soll nun zu Recht werden. Der Staat wird somit zum zentralen Akteur und Mittel der Landnahme. Legitimiert werden diese Vorstöße mit einem klassischen Modernisierungsnarrativ, einem unverhohlenen Rassismus gegen Indigene und Afrobrasilianer*innen und einer Abwertung ihrer landwirtschaftlichen Praktiken und Kulturen sowie der Kriminalisierung und „Rote-Socken“-Hetzjagd auf soziale Bewegungen, Umweltorganisationen und NGOs.

Der Ansatz der Landnahme als eine Variante der fortgesetzten ursprünglichen Akkumulation erwies sich als hilfreich. Konzeptionell und empirisch ist noch genauer zu klären, welche Rolle lokale Wissensordnungen bei der aktuellen Formierung von Widerständigkeiten spielt. Um den zeitdiagnostischen Anspruch des Konzepts zu stärken, wäre zu untersuchen, inwiefern global im Kontext des weltweiten Rechtsrucks eine neue Landnahme stattfindet.

Inwieweit die Landnahme der gesamten Amazonasregion am Ende gelingt oder scheitert, wie zuletzt in den 1980er Jahren, ist noch nicht absehbar. Denn diese Versuche der Landnahme bleiben nicht unwidersprochen, wie zahlreiche Proteste und Allianzen zwischen unterschiedlichen Gruppen in Brasilien und weltweit bereits verdeutlichen. In der Zusammenschau mit den anderen Beiträgen dieses Schwerpunktheftes wird deutlich, dass sie eine gemeinsame Vorstellungen des Sozialen haben: Gemeinschaft wird über die Anerkennung der Differenz und demokratischer Teilhabe aller gedacht. Diese transnationalen Allianzen müssen weiterhin vertieft werden - insbesondere zwischen sozialen und Umweltbewegungen. In den Worten von Brum (2020):

Noch heute ist es einem Teil der Gesellschaft und auch einigen Naturschützer*innen nicht klar, dass der Kampf für eine Agrarreform auch ein Kampf für Waldschutz ist. Ohne soziale Gerechtigkeit in Amazonien wird es auch keine Klimagerechtigkeit geben. (Brum 2020, S. 113, eigene Übersetzung)

Funding Open Access funding enabled and organized by Projekt DEAL.

Open Access Dieser Artikel wird unter der Creative Commons Namensnennung 4.0 International Lizenz veröffentlicht, welche die Nutzung, Vervielfältigung, Bearbeitung, Verbreitung und Wiedergabe in jeglichem Medium und Format erlaubt, sofern Sie den/die ursprünglichen Autor(en) und die Quelle ord- 
nungsgemäß nennen, einen Link zur Creative Commons Lizenz beifügen und angeben, ob Änderungen vorgenommen wurden.

Die in diesem Artikel enthaltenen Bilder und sonstiges Drittmaterial unterliegen ebenfalls der genannten Creative Commons Lizenz, sofern sich aus der Abbildungslegende nichts anderes ergibt. Sofern das betreffende Material nicht unter der genannten Creative Commons Lizenz steht und die betreffende Handlung nicht nach gesetzlichen Vorschriften erlaubt ist, ist für die oben aufgeführten Weiterverwendungen des Materials die Einwilligung des jeweiligen Rechteinhabers einzuholen.

Weitere Details zur Lizenz entnehmen Sie bitte der Lizenzinformation auf http://creativecommons.org/ licenses/by/4.0/deed.de.

\section{Literatur}

Abbrex, J. Jr (2005). "Terra sem povo", crime sem castigo. In M. G. Torres (Hrsg.), Amazônia revelada. Os descaminhos ao longo da BR-163 (S. 21-66). Brasília: CNPq.

de Abreu, C., \& Russau, C. (2020). Im Fadenkreuz von Bolsonaro. Bolsonaro-Regierung droht mit dem Ausstieg aus der ILO-Konvention 169, um industrielle Landwirtschaft, Bergbau und Wasserkraftnutzung in indigenen Territorien Brasiliens durchzusetzen. Freiburg: Kobra - Kooperation Brasilien. https://www.kooperation-brasilien.org/de/themen/landkonflikte-umwelt/im-fadenkreuzvon-bolsonaro. Zugegriffen: 5. September 2020.

Agarwal, B. (1998). The gender and environment debate. In R. Keil, D. V. J. Bell, P. Penz \& L. Fawcett (Hrsg.), Political ecology. Global and local. Innis centenary series. (S. 193-219). London, New York: Routledge.

Alessi, G. (2019). MP prevê que general "monitore" ONGs e exclui menção a LGBTs em pasta de Direitos Humanos. El País Brasil. https://brasil.elpais.com/brasil/2019/01/02/politica/1546454574_434358. html. Zugegriffen: 6. Sept. 2020.

d Almeida, A.W.B. (2008). Terras de quilombos, terra indígenas, 'babaçuais livres', "castanhais do povo", faxinais e fundos de pastos. Terras tradicionalmente ocupadas (2. Aufl.). Coleção Tradição \& ordenamento jurídico, Bd. 2. Manaus: Projeto Nova Cartografia Social da Amazôn.

d Almeida, A. W. B. (2010a). Amazônia a dimensão política dos conhecimentos tradicionais. In A. W. B. d Almeida (Hrsg.), Conhecimento tradicional e biodiversidade. Normas vigentes e propostas 2. Aufl. Documentos de bolso, (Bd. 4, S. 11-44). Manaus: PPGAS-UFAM; CESTU-UEA.

d Almeida, A. W.B. (2010b). Direitos territoriais e étnicos: as estratégias dos agronegócios na Amazônia. In A. Zhouri \& K. Laschefski (Hrsg.), Desenvolvimento e conflitos ambientais (S. 382-387). Belo Horizonte: Editora UFMG. https://doi.org/10.7476/9788542303063.0016.

Almeida, A. W. B., \& Carvalho, G. (Hrsg.). (2009). O plano IIRSA na visão da Sociedade Civil Pan-Amazônica. Belém: UFPA.

Alnasseri, S. (2003). Ursprüngliche Akkumulation, Artikulation und Regulation. Aspekte einer globalen Theorie der Regulation. In U. Brand \& W. Raza (Hrsg.), Fit für den Postfordismus? Theoretisch-politische Perspektiven des Regulationsansatzes (1. Aufl. S. 131-157). Münster: Westfälisches Dampfboot.

Assunção, M.R. (1993). Pflanzer, Sklaven und Kleinbauern in der brasilianischen Provinz Maranhão 1800-1850. Berliner Lateinamerika-Forschungen, Bd. 2. Frankfurt am Main: Vervuert. https://doi. org/10.31819/9783954879984.

Backhouse, M. (2015). Grüne Landnahme - Palmölexpansion und Landkonflikte in Amazonien. Münster: Westfälisches Dampfboot.

Brito, B., \& Barreto, P. (2009). Os riscos e os princípios para a regularização fundiária na Amazônia. Belém: Imazon.

Bröckelmann-Simon, M. (1994). Landlose in Brasilien. Entstehungsbedingungen, Dynamik und Demokratisierungspotential der brasilianischen Landlosenbewegung. Mettingen: Brasilienkunde-Verlag.

Browder, J., Pedlowski, M.A., \& Walker, R. (2008). Revisiting theories of frontier expansion in the Brazilian Amazon: a survey of the colonist farming population in rondo nia's post-frontier, 1992-2002 . World Development, 36(8), 1469-1492. https://doi.org/10.1016/j.worlddev.2007.08.008.

Brum, E. (2020). A miliciarização da Amazônia: como o crime vira lei e o criminoso "cidadão de bem" na maior floresta tropical do mundo. In CPT (Hrsg.), Conflitos no Campo Brasil 2019 (S. 105-115). Goiânia: CPT. 
Bunk, B. (2018). Bildung und soziale Bewegung. Kultur und Bildung, Bd. 6. Paderborn: Schöningh. https:// doi.org/10.30965/9783657772520.

Canuto, A., Cruzeiro, M. A., dos Santos, P. C. M., \& de Siqueira, R. A. (2020). Conflitos por Terra em 2019, uma introdução. In CPT (Hrsg.), Conflitos no Campo Brasil 2019 (S. 100-104). Goiânia: CPT.

Cleary, D. (1993). After the frontier. Problems with political economy in the modern Brazilian Amazon. Journal of Latin American Studies, 25(2), 331-349. https://doi.org/10.1017/S0022216X00004685.

Coy, M. (1988). Regionalentwicklung und regionale Entwicklungsplanung an der Peripherie in Amazonien. Probleme und Interessenkonflikte bei der Erschließung einer jungen Pionierfront am Beispiel des brasilianischen Bundesstaates Rondônia. Tübinger Beiträge zur geographischen LateinamerikaForschung, Bd. 5. Tübingen: Universität Tübingen.

CPT (2020). Conflitos no Campo Brasil 2019. Goiânia: CPT.

De Angelis, M. (2001). The continuous character of capital's "enclosures". The Commoner (2), 1-22. http://www.commoner.org.uk/02deangelis.pdf. Zugegriffen: 29. Mai 2013.

Dörre, K. (2009). Die neue Landnahme. Dynamiken und Grenzen des Finanzmarktkapitalismus. In K. Dörre, S. Lessenich \& H. Rosa (Hrsg.), Soziologie - Kapitalismus - Kritik. Eine Debatte (S. 21-86). Frankfurt am Main: Suhrkamp.

Escobar, A. (1996). Construction nature. Elements for a post-structuralist political ecology. Futures, 28(4), 325-343. https://doi.org/10.1016/0016-3287(96)00011-0.

Escobar, H., Dorado, \& Moisés (2020). Desmatamento da Amazônia dispara de novo em 2020. Dados de satélite revelam um aumento de $34 \%$ na taxa de derrubada da floresta nos últimos 12 meses. Journal da USP. https://jornal.usp.br/ciencias/desmatamento-da-amazonia-dispara-de-novo-em-2020/. Zugegriffen: 5. Sept. 2020.

Fanon, F. (1981). Die Verdammten dieser Erde (1. Aufl.). Bd. 668. Frankfurt am Main: Suhrkamp.

Foweraker, J. (1981). The struggle for land. A political economy of the pioneer frontier in Brazil from 1930 to the present day. Cambridge: Cambridge University Press. https://doi.org/10.1017/ CBO9780511572258.

Görg, C. (2003). Regulation der Naturverhältnisse. Zu einer kritischen Theorie der ökologischen Krise. Münster: Westfälisches Dampfboot.

Görg, C. (2004). Inwertsetzung. In Historisch-kritisches Wörterbuch des Marxismus (Bd. 6, S. 1501-1506). Hamburg: Argument.

Greenpeace (2020). Greenpeace verurteilt drohendes Landraub-Gesetz im Amazonas. https://presse. greenpeace.at/greenpeace-verurteilt-drohendes-landraub-gesetz-im-amazonas/. Zugegriffen: 6. Sept. 2020.

Haraway, D. (1988). Situated nnowledges: the science question in feminism and the privilege of partial perspective. Feminist Studies, 14(3), 575-599. https://doi.org/10.2307/3178066.

Harvey, D. (2005). Der neue Imperialismus. Hamburg: VSA.

ISA (2019). Assessor da bancada ruralista é nomeado presidente da Funai. Instituto Socioamtiental. São Paulo. https://www.socioambiental.org/pt-br/noticias-socioambientais/assessor-da-bancadaruralista-e-nomeado-presidente-da-funai. Zugegriffen: 6. Sept. 2020.

Jürgens, G. (2020). Indigene in Gefahr. https://www.greenpeace.de/themen/waelder/waldnutzung. Zugegriffen: 6. Sept. 2020.

Kößler, R. (2013). Prozesse der Trennung - Gewalt im Ursprung und fortgesetztes Prozessieren des Kapitals. In M. Backhouse, O. Gerlach, S. Kalmring \& A. Nowak (Hrsg.), Die globale Einhegung - Krise, ursprüngliche Akkumulation und Landnahmen im Kapitalismus (S. 20-39). Münster: Westfälisches Dampfboot.

Lutz, B. (1984). Der kurze Traum immerwährender Prosperität. Eine Neuinterpretation der industriellkapitalistischen Entwicklung im Europa des 20. Jahrhunderts. Frankfurt: Campus.

Luxemburg, R. (1923). Die Akkumulation des Kapitals. Ein Beitrag zur ökonomischen Erklärung des Imperialismus. Berlin: Vereinigung internationaler Verlags-Anstalten.

Maerba, J., Milanez, B., \& Wnaderley, L. J. (Hrsg.). (2012). Novo Marco legal da Mineração no Brasil: para quê? Para quem? Rio de Janeiro: FASE.

de Martins, J.S. (2009). Fronteira. A Degradação do Outro nos Confins do Humano. São Paulo: Editora Contexto.

Marx, K. (2005). Das Kapital. Erster Band. Der Produktionsprozess des Kapitals. In Marx Engels Werke $(M E W)$ Bd. 23. Berlin: Dietz-Verlag.

Meillassoux, C. (1978). Die wilden Früchte der Frau. Über häusliche Produktion und kapitalistische Wirtschaft (2. Aufl.). Frankfurt am Main: Syndikat. https://doi.org/10.5771/0506-7286-1978-3-381. 
Neuburger, M. (2002). Pionierfrontentwicklung im Hinterland von Cáceres (Mato Grosso, Brasilien). Ökologische Degradierung, Verwundbarkeit und kleinbäuerliche Überlebensstrategien. Tübinger Beiträge zur geographischen Lateinamerika-Forschung, Bd. 23. Tübingen: Universität Tübingen.

de Oliveira, A. U. (2005). BR-163 Cuiabá-Santarém. Geopolítica, grilagem, Violência e mundialização. In M. G. Torres (Hrsg.), Amazônia revelada. Os descaminhos ao longo da BR-163 (S. 67-184). Brasília: CNPq.

Oxfam (2019). Menos de $1 \%$ das propriedades agrícolas é dona de quase metade da área rural brasileira. https://www.oxfam.org.br/publicacao/menos-de-1-das-propriedades-agricolas-e-dona-de-quasemetade-da-area-rural-brasileira/. Zugegriffen: 6. Sept. 2020.

Peters, B. (2019). Macron hat einen dummen Fehler gemacht. Süddeutsche Zeitung. https://www. sueddeutsche.de/politik/amazonas-macron-bolsonaro-kolonialismus-1.4573626. Zugegriffen: 3 . Sept. 2020.

Polanyi, K. (1978). The great transformation. Politische und ökonomische Ursprünge von Gesellschaften und Wirtschaftssystemen (1. Aufl.). Frankfurt/Main: Suhrkamp.

Quijano, A.E. (Hrsg.). (2016). Kolonialität der Macht, Eurozentrismus und Lateinamerika. Bd. 17. Wien: Turia + Kant

Russau, C. (2020). Ehrenhuldigung für Foltergeneral. Freiburg: Kobra - Kooperation Brasilien. https:// www.kooperation-brasilien.org/de/ehrenhuldigung-fuer-foltergeneral. Zugegriffen: 6. Sept. 2020.

Schenk, M., \& Kliver, C. (2020). Linke will Angriffe auf Landarbeiter in Brasilien im Bundestag thematisieren. amerika21. https://amerika21.de/2020/09/243193/brasilen-deutschland-bundestag-landlose. Zugegriffen: 6. Sept. 2020.

Schmink, M.C., \& Wood, C.H. (1992). Contested frontiers in Amazonia. New York: Columbia Univ. Pr.

Schönenberg, R. (1993). Konflikte und Konfliktregulation in Amazonien. Ursachen, Formen und Folgen ländlicher Konflikte in Süd-Pará. Bd. 74. Bonn: Holos.

de Souza, O.B. (2016). O que o governo Dilma fez (e não fez) pela reforma agrária?, ISA. https:// www.socioambiental.org/pt-br/noticias-socioambientais/o-que-o-governo-dilma-fez-e-nao-fezpela-reforma-agraria. Zugegriffen: 6. Sept. 2020.

Stedil, J.P., \& Fernandes, B. M. (1999). Brava gente: a trajetória do MST e a luta pela terra no Brasil. São Paulo: Fundação Perseu Abramo.

Torres, M. (2005). Fronteira, um eco sem fim. Considerações sobre a ausência do Estado e exclusão social nos municípios paraenses do eixo da BR-163. In M. G. Torres (Hrsg.), Amazônia revelada. Os descaminhos ao longo da BR-163 (S. 271-319). Brasília: CNPq.

Torres, M. (2018). Grilagem para Principiantes: Guia de Procedimentos Básicos para o Roubo de Terras Públicas. In M. I. M. Marques, C. I. Bernini, L. Cavalieri, P.C. R. Perez, E. Castro \& A. Cornetta, et al. (Hrsg.), Perspectivas de natureza 1. Aufl. Geografias, Formas De Natureza e Politica. (S. 285-314). São Paulo: Annablume.

Torres, M., \& Branford, S. (2018). Amazon Besieged. By dams, soya, agribusiness and land-grabbing. Warwickshire: Practical Action Publishing. https://doi.org/10.3362/9781909014091.

Torres, M., Doblas, J., \& Alarcon, D. F. (2017). Dono é quem desmata. Conexões entre grilagem e desmatamento no sudoeste paraense. Altamira: Instituto Agronômico da Amazônia; Urutu-branco.

Treccani, G.D. (1998). Violência e Grilagem: Instrumentos de Aquisição da Propriedade da Terra no Pará. Belém: UFPA.

Velho, O. G. (1979). Capitalismo Autoritário e Campesinato. Um estudo comparativo a partir da fronteira em movimento (2. Aufl.). São Paulo: Difel.

Wallerstein, I. (1986). Das moderne Weltsystem. Frankfurt am Main: Syndikat.

von Werlhof, C., Mies, M., \& Bennholdt-Thomsen, V. (1988). Frauen, die letzte Kolonie. Zur Hausfrauisierung der Arbeit. Reinbek bei Hamburg: Rowohlt.

Whitehead, J. (2016). Intersectionality and primary accumulation. Monthly Review 68 (6), o.S. https:// monthlyreview.org/2016/11/01/intersectionality-and-primary-accumulation/. Zugegriffen: 18. Juni 2017. https://doi.org/10.14452/MR-068-06-2016-10_3.

Wood, C.H. (1983). Peasant and capitalist production in the Brazilian Amazon: a conceptual framework for the study of frontier expansion. In E. F. Morán (Hrsg.), The dilemma of Amazonian development (S. 259-277). Boulder: Westview Press. https://doi.org/10.4324/9780429310041-13. 